\title{
Reaction norms of Arabidopsis. IV. Relationships between plasticity and fitness
}

\author{
MASSIMO PIGLIUCCI* \& CARL D. SCHLICHTING \\ Department of Ecology and Evolutionary Biology, University of Connecticut, Storrs, CT 06269, U.S.A.
}

\begin{abstract}
The study of the association between fitness and reaction norms is of primary importance given the hypothesized role for phenotypic plasticity in shaping evolutionary patterns: in microevolution, as one mechanism for maintaining genetic variation, and in macroevolution, as a means of generating phenotypic novelties. In a glasshouse experiment, we investigated variation in reaction norms to nutrient availability in populations of Arabidopsis thaliana, and the relationship between this variation and reproductive fitness. We found evidence for acrosstreatment directional selection on the means for leaf number, flowering time, plant height, branching and growth rate; across-treatment stabilizing selection was detected for growth rate; and across-treatment disruptive selection was significant for leaf number. We also uncovered selection on the plasticity of some traits: directional for the plasticity of branching, and stabilizing for the plasticity of both branching and growth rate. When the two environments were considered separately, directional selection for height was detected under low nutrients; under high nutrients, we found evidence for directional selection on leaf number and height, and for disruptive selection on flowering time. The genetic correlation between a trait's expression in one environment and its expression in the alternate environment was positive and highly significant only for flowering time and growth rate. A principal components analysis revealed possible constraints on future selection responses, because of correlations among character means and among character plasticities.
\end{abstract}

Keywords: Arabidopsis thaliana, evolution, fitness, phenotypic plasticity.

\section{Introduction}

Phenotypic plasticity is the ability of an organism to produce different phenotypes in response to different environments (Schmalhausen, 1949). It is under genetic control (Bradshaw, 1965; Schlichting, 1986; Scheiner, 1993), and is a potential target for selection when it plays a role in the adaptation of organisms to changing environmental conditions (Sultan, 1987; Schlichting \& Pigliucci, 1993, 1995). The relationship between character plasticity and fitness is therefore of substantial importance for our understanding of how changes in the reaction norm may evolve (Levins, 1963; Via, 1993; Weis \& Gorman, 1990). Few studies, however, have attempted to quantify directly this relationship (e.g. Lacey et al., 1983; Weis \& Gorman, 1990).

\footnotetext{
*Correspondence: Department of Botany, University of
} Tennessee, Knoxville, TN 37996-1100, U.S.A.
There are several reasons for this unsatisfactory state of affairs, not the least being the conceptual and computational problems related to estimations of fitness (see Ollason, 1991 for a discussion and references; de Jong, 1994). Functional ecological studies of fitness under controlled conditions run into problems such as those pointed out by Service \& Rose (1985): the genetic architecture (i.e. the genetic relationships among a series of characters, including fitness) may be altered when an organism is exposed to a different or novel environment. This means that special care must be taken in the choice of environmental variables. For phenotypic plasticity in particular, we need to determine under what conditions selection acts on plasticity per se (Bradshaw, 1965; Schlichting \& Pigliucci 1993, 1995), as opposed to on the within-environment character expression (Via 1993).

We use the annual herbaceous plant Arabidopsis thaliana (Brassicaceae) to investigate the relationships between fitness and the reaction norms of 
several characters to nutrient availability. The choice of this experimental system was based on the following points. (1) In a weedy annual like $A$. thaliana, a major component of fitness is the total seed output at the end of the growing season; this is relatively easy to measure in a large number of individuals. As an annual, the total fruit output is an excellent and easy to use approximation to seed production and establishment, because of the very tight and relatively environmentally-invariant correlation between fruit and seed production, and very high germination rates under a wide range of conditions (Westerman \& Lawrence, 1971). (2) There is evidence that $A$. thaliana is subject to regular fluctuations in nutrient availability under field conditions. Thompson (1994) showed that $A$. thaliana can find itself in the presence of high concentrations of nutrients if it germinates not long after the mother plant has decayed; otherwise, the plant can experience an environment characterized by very low nutrient availability. This fluctuation has profound effects on individual phenotypes and on population dynamics. (3) There is substantial variation among populations in reaction norms to nutrient levels in $A$. thaliana (Pigliucci \& Schlichting, 1995), suggesting the possibility of an effective response to natural selection, if comparable variation exists within populations.

The previous three papers of this series have investigated other aspects of reaction norms variation in A. thaliana. Pigliucci et al. (1995a) demonstrated genetic differentiation among populations for the response to a broad range of three fundamental abiotic parameters: water, light and nutrients. They also showed, through the use of path analysis, both genetic and environmentally-induced variation in character correlations. Pigliucci et al. (1995b) explored the environment-dependent expression of genetic variation in $A$. thaliana and found that more variation was expressed in 'stressful' vs. benign environments and in a set of unordered environments compared with gradients. Finally, Pigliucci \& Schlichting (1995) looked specifically at the response to nutrients availability in a survey of worldwide populations of $A$. thaliana.

In this study we address the following questions. (i) Is there genetic variation within populations for the reaction norms to nutrients availability for genotypes from natural populations of $A$. thaliana, and how does this compare with the magnitude of among-population variation? Because genetic variation for plasticity is a prerequisite for any future response to selective pressures, we will assess its presence among 12 families from each of three natural populations and an isogenic line (see below) for reaction norms of several characters in response to two levels of nutrients. (ii) What are the relationships between reaction norms of major phenotypic traits to nutrients and fitness in $A$. thaliana? We address this question by comparing reaction norms of genotypes with different total reproductive output and by regression analyses of reaction norm parameters on fruit production.

\section{Materials and methods}

\section{Plant material and experimental set-up}

We used 12 families of plants for each of the following three populations (so-called 'ecotypes') of Arabidopsis thaliana (L.) Heynh., an annual, highly selfing weed (Abbott \& Gomes, 1989): Greenville (henceforth, G), Kendalville (K), and Turk Lane (T). We also included the isogenic line Landsberg (L) for comparison with other studies on the population biology and genetics of this species. The families were obtained by selfing plants grown from seeds procured from the Crucifer Genetics Cooperative (CGC) at the University of Wisconsin-Madison. Population $\mathrm{K}$ and isogenic line $\mathrm{L}$ represented early flowering populations, whereas populations $\mathrm{G}$ and $\mathrm{T}$ are classified as late flowering. Flowering time is of major ecological importance in this species (Jones, 1971; Westerman \& Lawrence 1971), and it is therefore used as an indicator of the existence of local races.

Seeds were spread on filter paper in petri dishes in a cold room for 2 days to help synchronize germination. They were then transferred for 2 days to ambient temperature and imbibed with water to induce germination. Plants were grown in the greenhouses of the University of Connecticut at Storrs, in $5 \mathrm{~cm}$ pots. The experiment included three populations, 12 families per population, two treatments, and three replicates per treatment per family, plus the same number of replicates of Landsberg. The two treatments differed in the concentration of available nitrogen, phosphorus and potassium ( 0 vs. 3 pellets of $14: 14: 14 \mathrm{~N}: \mathrm{P}: \mathrm{K}$ Osmocote, Slater Supply). The low nutrients level represents a stress, similar to conditions presumably encountered by natural populations on very dry and exposed soil; the higher level allows vigorous growth, similar to natural populations living in rich and undisturbed environments (M. Pigliucci, personal observation). As Thompson (1994) did not report any quantitative assessment of nutrients abundance, we did not make any attempt to match explicitly those conditions. Plants were grouped in two blocks, with treatments 
and replicates randomized within blocks (one or two per block). Plants were grown in a small area, in standard germinating soil mix, and watered daily. The low mortality was randomly distributed with respect to family, but slightly higher in low nutrients.

The following characters representing important aspects of the $A$. thaliana phenotype (Pigliucci et al., 1995a,b) were scored: (i) number of leaves (vegetative growth); (ii) flowering time (phenology/developmental timing); (iii) final height of the main stem (size); (iv) number of lateral branches on the main stem (architecture); and (v) growth rate (total height of the stem divided by the difference between senescence time and bolting time). Reproductive fitness was measured as the total number of fruits produced by the plant at senescence.

\section{Data analysis}

Genetic variation for reaction norms to nutrients availability We performed a series of mixed-model analyses of variance (procedure GLM in SAS, 1990) assessing the influence of the following sources of variation on each character: Treatment (i.e. phenotypic plasticity); Population (i.e. genetic variation across treatments among populations and the isogenic line); Family nested within Population (i.e. genetic variation across treatments within the three populations); Treatment by Population interaction (i.e. genetic variation for plasticity among populations and the isogenic line); and Treatment by Family interaction (i.e. genetic variation for plasticity within populations). Block effects, included in the initial model, were not significant and were not included in the final model. Both Population and Treatment were considered fixed effects. Families were considered random effects and therefore the Population effect was tested over a combination of error and Family variances. Significance levels were determined by a sequential Bonferroni procedure to compensate for simultaneous comparisons involving the five traits.

We also graphically inspected the phenotypic scatter of the different families in the two treatments. Instead of plotting the standard reaction norms (i.e. functions describing the behaviour of each family in a Treatment vs. Phenotype diagram), we graphed the position of each family in the phase space defined by its phenotype under low nutrients (on the $x$-axis) and its phenotype under high nutrients (on the $y$-axis). Each family is then represented by a single point identified by a pair of coordinates. When the two axes are plotted on the same scale, this graph presents several interesting properties. A family lying on the main diagonal will have equal phenotypic values in both environments (i.e. a flat reaction norm). The perpendicular diagonal represents a continuum between the two possible patterns of increasing plasticity: a much higher phenotypic value in one environment than in the other (moving toward the upper left or lower right corners of the diagram). The across-environment average phenotype remains constant along this axis. The intersection of these diagonals represents a case of no plasticity and an average phenotype in both environments (scaled with respect to the phenotypic range actually observed). The univariate reaction norm plots were also used to help visualize the adaptive landscape. The relative fitness of the families was subdivided into three equal classes: below average fitness (represented by minus signs), average (circles), and above average (plus signs).

The correlation between the two axes is an estimate of the genetic correlation between the expression of a trait under low vs. high nutrients. We used the family means, instead of the variance components, to calculate these genetic correlations, although estimates based on family means are known to be biased upwards because of the inclusion of maternal effects (Roff \& Preziosi, 1994). Our choice was based on two considerations: first, seed size and number are relatively constant and families originated from maternal plants grown under uniform conditions, making it unlikely that the two estimates will differ greatly in magnitude (see Geber, 1990). Secondly, although estimates based on variance component analysis are more precise (i.e. less biased), they are far less accurate (i.e. associated with a higher error variance; Shaw, 1991). We therefore opted for a likely smaller bias in the precision of our estimates but a sufficient degree of accuracy to allow tests of significance.

\section{Relationships between reaction norms and reproductive} fitness Reproductive fitness of families was regressed against the linear and quadratic terms (Rausher, 1992) in two separate analyses (see Weis \& Gorman, 1990) for both the across-treatment mean phenotype and the plasticity (i.e. the difference in phenotype between high and low nutrients). The linear terms quantify the intensity and sign of directional selection, whereas the quadratic terms assess stabilizing or disruptive selection. We used the procedure REG in SAS (1990), and calculated standardized regression coefficients, which are easier to interpret than the unstandardized ones (Weis \& Gorman, 1990). We analysed each trait separately because the full model (all linear and quadratic 
effects of trait means and plasticities) was intractable.

An analysis on the descriptors of the reaction norm (i.e. elevation and slope) tests for selection directly on plasticity or across-environment response, but also assumes that the environments are equally frequent. Although true for our controlled conditions, this might not hold for natural populations, where both environmental frequency and the number of individuals per environment can vary. For this reason, we also performed regression analyses of the relationship between all traits and reproductive fitness within each of the two treatments to help in making predictions about selection regimes on reaction norms if the environments were not equiprobable. Note that in each case where we found significant quadratic effects we classified the type of selection (stabilizing or disruptive) on the basis of the sign of the quadratic coefficient and of plots of partial residuals (not shown).

The univariate analysis described so far does not account for potential correlations among averages or plasticities of the same or different traits. To gain a multivariate perspective of the reaction norms we carried out a principal components analysis on the genotypic means, using the characters' averages and plasticities as variables. We then performed regression analyses of the first two principal components scores on reproductive fitness.

\section{Results}

Variability in reaction norms to nutrient availability

The first question we addressed concerned the variability in the elevation (i.e. across-environment mean) and slope (i.e. plasticity) within and among populations of $A$. thaliana. The analysis of variance (Table 1) shows a significant Treatment effect (phenotypic plasticity) for height and number of branches: the overall (across families) values of these characters tended to increase with availability of nutrients. The number of leaves in the rosette, time to flowering and growth rate remained stable across environments. The three populations and the isogenic line showed very marked differentiation for all traits included in the analysis (significant Population effect in the ANOVAS). Four characters (all except growth rate) were also significantly different among families within natural populations. There was significant genetic variation for plasticity in flowering time (among populations), and for the number of branches (among families) (Table 1).

The phenotypic phase space diagrams (Fig. 1a-e) allow us to visualize the actual scatter of families according to characteristics of their reaction norms. The number of leaves in the rosette ranged from fewer than 10 to around 200, with a concentration of families characterized by reaction norms with leaf

Table 1 Analysis of variance of five traits in three populations and one isogenic line of Arabidopsis thaliana

\begin{tabular}{lccccc}
\hline $\begin{array}{l}\text { Source } \\
\text { (d.f.) }\end{array}$ & Leaves & $\begin{array}{c}\text { Flowering } \\
\text { time }\end{array}$ & Height & Branches & $\begin{array}{c}\text { Growth } \\
\text { rate }\end{array}$ \\
\hline Treatment & 4154.02 & 15.95 & $\mathbf{4 1 4 2 3 . 1 4}$ & $\mathbf{1 0 3 . 6 7}$ & 11.57 \\
$(1)$ & $(0.1804)$ & $(0.1991)$ & $(0.0084)$ & $(0.0001)$ & $(0.8488)$ \\
Population & $\mathbf{3 2 7 9 7 . 3 4}$ & $\mathbf{2 8 7 9 . 1 4}$ & $\mathbf{7 7 7 8 9 8 . 5 7}$ & $\mathbf{1 4 6 . 7 0}$ & $\mathbf{2 1 2 2 5 . 2 9}$ \\
(3) & $(0.0010)$ & $(0.0001)$ & $(0.0001)$ & $(0.0005)$ & $(0.0001)$ \\
Family (Pop) & $\mathbf{4 8 6 5 . 9 9}$ & $\mathbf{9 0 . 3 1}$ & $\mathbf{2 9 4 1 7 . 8 4}$ & $\mathbf{1 9 . 5 0}$ & 478.44 \\
(33) & $(0.0018)$ & $(0.0001)$ & $(0.0001)$ & $(0.0001)$ & $(0.0577)$ \\
Treat *Pop & 1877.45 & $\mathbf{3 3 . 1 0}$ & 630.93 & 1.14 & 305.20 \\
(3) & $(0.4849)$ & $(0.0187)$ & $(0.9543)$ & $(0.8317)$ & $(0.4126)$ \\
Treat *Family & 2520.47 & 8.48 & 7042.57 & $\mathbf{1 2 . 2 5}$ & 200.22 \\
(33) & $(0.3442)$ & $(0.6438)$ & $(0.2160)$ & $(0.0001)$ & $(0.9348)$ \\
Error & 2286.91 & 9.56 & 5752.32 & 3.93 & 316.78 \\
(114) & & & & &
\end{tabular}

Type III MS are reported. Families within Populations were considered random effects and therefore the Population effect was tested on the Family and error variances; all other effects were tested over the error variance.

Boldface indicates effects significant after a sequential Bonferroni correction (across traits), which accounts for multiple simultaneous tests.

$P$ values are in parentheses below the corresponding MS. 


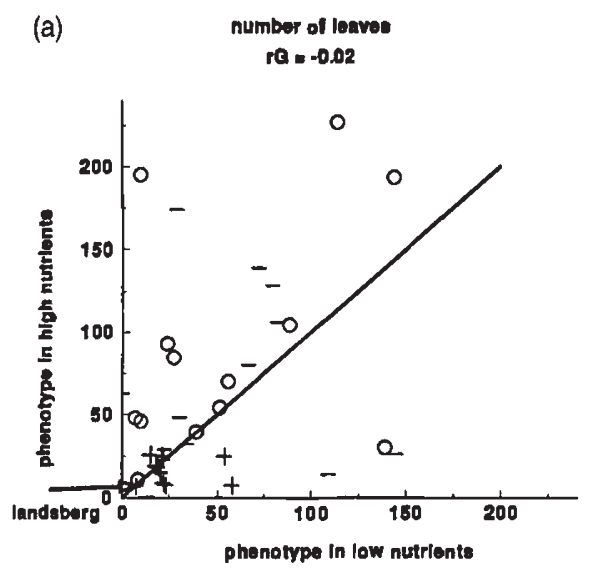

(b)

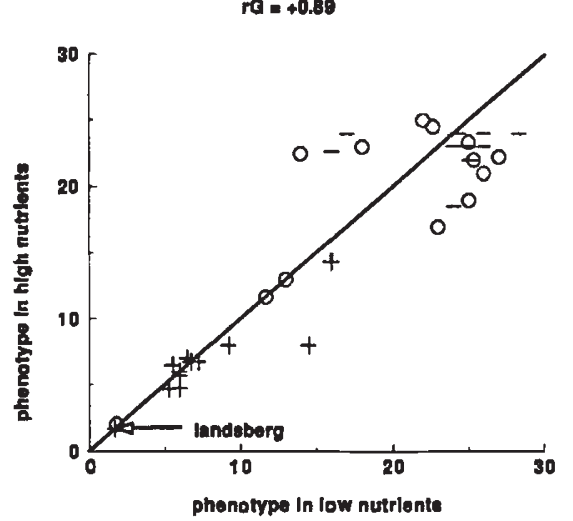

(c)

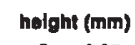

hoight (mm)

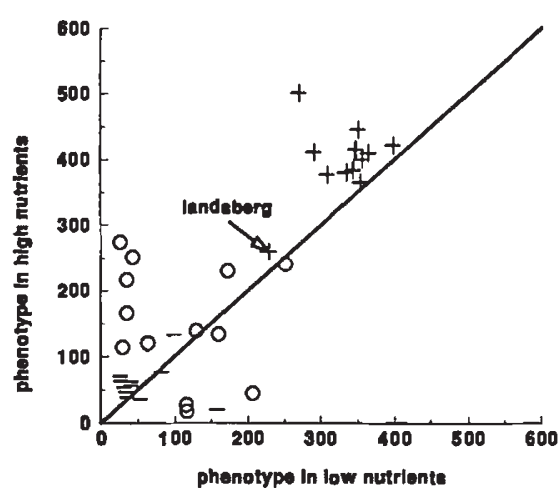

(d)

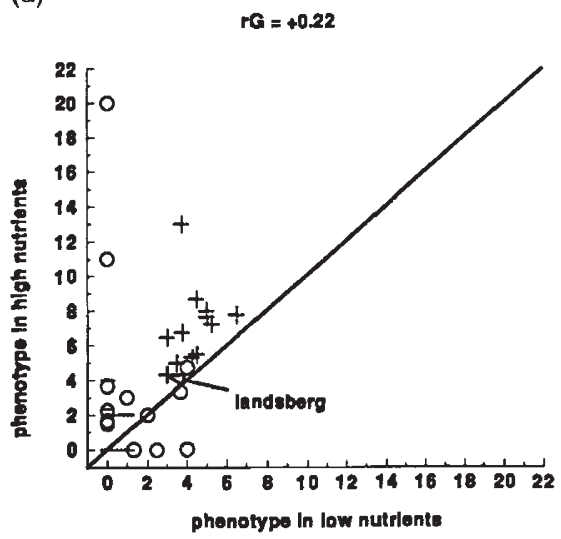

(e)
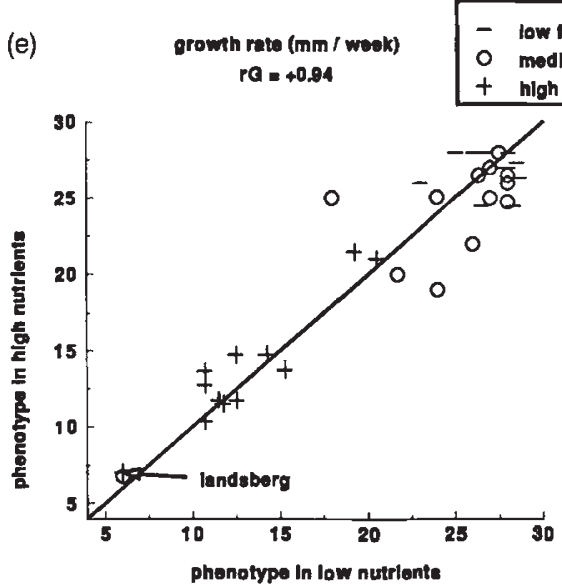

Fig. 1 Plot of reaction norms of 37 families of Arabidopsis thaliana in (low nutrients vs. high nutrients) phase space. Symbols in the legend indicate low (minuses), medium (circles), or high (pluses) levels of across-environment fitness. (a) number of leaves; (b) flowering time; (c) height; (d) number of branches; (e) growth rate. The main diagonal represents the line of no plasticity on each diagram, not the results of a regression analysis. The genetic correlation between the expressions of each trait in the two environments is reported.

numbers below 50 in either treatment (Fig. 1a). Landsberg showed the lowest number of leaves in either environment. The genetic correlation between the expression of this trait in the two treatments was close to zero (i.e. leaf production of a family in one environment is not a predictor of leaf production in the other environment). Flowering time varied from about two to almost 25 weeks, with Landsberg flowering fastest in both environments. The scatter away from the main diagonal was markedly reduced (compared with leaf number), as is evident from a significant genetic correlation between environments of +0.89 . The families seem to fall into two main clusters, one flowering within 10 weeks, the other after 20, with a few families in between (Fig. 1b). The range for height was between 50 and $500 \mathrm{~mm}$, with two main clusters (less than 200 and more than $300 \mathrm{~mm}$ ), and a tendency for families to fall well above the main diagonal (i.e. to produce taller individuals under high nutrients). The genetic correlation between the phenotypic expression in the two treatments was not significant $(+0.35)$. Landsberg was of intermediate height in both environments (Fig. 1c). The distribution of number of branches among the 37 families varied from zero to 20 , with a marked propensity to produce more branches under the high nutrient treatment. The genetic correlation was not significant $(+0.22)$, and Landsberg produced an intermediate number of branches in both environments (Fig. 1d). Finally, the scatter around the main diagonal of reaction norms for growth rate was very limited, with most families 
Table 2 Multiple regressions relating the across-environment average and plasticity of each trait in Arabidopsis thaliana to across-environment fitness

\begin{tabular}{|c|c|c|c|c|}
\hline Trait & Variable & Regression coefficient & $t$-ratio & $P$ value \\
\hline \multirow[t]{4}{*}{ No. of leaves } & Mean, linear & -0.4977 & -3.131 & 0.0036 \\
\hline & Plasticity, linear & -0.0495 & -0.312 & 0.7573 \\
\hline & Mean, quadratic & +1.2483 & +2.697 & 0.0111 \\
\hline & Plasticity, quadratic & +0.0488 & +0.283 & 0.7788 \\
\hline \multirow[t]{4}{*}{ Flowering time } & Mean, linear & -0.8502 & -8.909 & 0.0001 \\
\hline & Plasticity, linear & -0.1209 & -1.267 & 0.2139 \\
\hline & Mean, quadratic & -0.6174 & -0.948 & 0.3505 \\
\hline & Plasticity, quadratic & -0.0375 & -0.324 & 0.7482 \\
\hline \multirow[t]{4}{*}{ Height } & Mean, linear & +0.9648 & +17.565 & 0.0001 \\
\hline & Plasticity, linear & -0.0491 & -0.894 & 0.3777 \\
\hline & Mean, quadratic & +0.6518 & +2.114 & 0.0424 \\
\hline & Plasticity, quadratic & +0.0373 & +0.570 & 0.5726 \\
\hline \multirow[t]{4}{*}{ No. of Branches } & Mean, linear & +1.1984 & +12.804 & 0.0001 \\
\hline & Plasticity, linear & -0.6103 & -6.520 & 0.0001 \\
\hline & Mean, quadratic & +0.6194 & +2.479 & 0.0186 \\
\hline & Plasticity, quadratic & -0.6855 & -4.898 & 0.0001 \\
\hline \multirow[t]{4}{*}{ Growth } & Mean, linear & -0.7863 & -7.648 & 0.0001 \\
\hline & Plasticity, linear & +0.0925 & +0.900 & 0.3746 \\
\hline & Mean, quadratic & -2.7910 & -4.143 & 0.0002 \\
\hline & Plasticity, quadratic & -0.2549 & -2.827 & 0.0080 \\
\hline
\end{tabular}

The analyses were carried out separately for each trait, and each included linear terms for across-environment mean and plasticity, and quadratic terms for mean and plasticity.

Standardized regression coefficients are reported.

Boldface indicates significant coefficients according to a sequential Bonferroni correction that accounts for multiple simultaneous tests across traits.

falling along the diagonal; the genetic correlation of +0.94 across environments is highly significant. Landsberg had the slowest growth rate under both conditions.

\section{Relationships between reaction norms and fitness}

The multiple regressions of parameters describing the reaction norms to nutrients on the average reproductive fitness are presented in Tables 2 and 3 . Across environments, we detected significant directional selection for decreased number of leaves, as well as significant disruptive selection on the same character. The corresponding plot in Fig. 1a visualizes this situation: the families characterized by the highest fitness are found in the lower left corner of the graph (i.e. they have low average numbers of leaves), and although they are almost all close to the main diagonal (indicating lack of plasticity), some produce a higher number of leaves under low nutrients. The secondary peak (i.e. evidence for disruptive selection) may arise from the relatively higher fitness of several families that produced large numbers of leaves in both environments or only in the high nutrients one (Fig. 1a, top centre and top left). We found a highly significant regression coefficient for the linear component of flowering time, indicating selection for early flowering among the families studied. In Fig. 1b most families with high fitness are concentrated in the area of no plasticity and early flowering. The regression analyses revealed significant directional selection for an increase in plant height; Fig. 1c shows that highly fit families are tall, with a tendency for them to be taller under high nutrients. Three of the four terms in the regression analysis of number of branches were highly significant, indicating simultaneous directional selection for increased branching and reduced plasticity of branching, as well as stabilizing selection on the plasticity. Therefore, high branching is favoured, but there seems to be selection against extreme plasticity (i.e. against production of branches only in the high nutrients treatment). In Fig. 1d the highest level of fitness is achieved by plants that branch the most, especially under high nutrients. Finally, we detected very significant directional 
Table 3 Multiple regressions relating the within-environment trait means to within-environment fitness of Arabidopsis thaliana

\begin{tabular}{llccr}
\hline Trait & Variable & Regression coefficient & $t$-ratio & $P$ value \\
\hline Low nutrients & & & & -1.845 \\
$\quad$ No. of leaves & Linear & -0.0746 & +1.156 & 0.0746 \\
Flowering time & Linear & +0.3091 & +2.390 & 0.2564 \\
Height & Linear & $+\mathbf{0 . 6 3 1 3}$ & +1.810 & 0.0231 \\
No. of branches & Linear & +0.3867 & -0.659 & 0.5149 \\
Growth rate & Linear & -0.1660 & -0.436 & 0.6661 \\
No. of leaves & Quadratic & -0.1373 & -0.122 & 0.9036 \\
Flowering time & Quadratic & -0.1644 & -0.819 & 0.4204 \\
Height & Quadratic & -0.6761 & +2.136 & 0.0423 \\
No. of branches & Quadratic & +0.9822 & -0.265 & 0.7931 \\
Growth rate & Quadratic & -0.3636 & -3.517 & 0.0014 \\
High nutrients & Linear & $-\mathbf{0 . 1 8 8 9}$ & +0.209 & 0.8357 \\
No. of leaves & Linear & +0.0572 & +6.844 & 0.0001 \\
Flowering time & Linear & $+\mathbf{0 . 9 8 4 9}$ & -0.817 & 0.4202 \\
Height & Linear & -0.0645 & -0.001 & 0.9990 \\
No. of branches & Linear & -0.0003 & -0.807 & 0.4270 \\
Growth rate & Quadratic & -0.1547 & +2.616 & 0.0146 \\
No. of leaves & Quadratic & $+\mathbf{1 . 6 9 4 2}$ & +1.566 & 0.1294 \\
Flowering time & Quadratic & +0.4809 & -1.375 & 0.1810 \\
Height & Quadratic & -0.2458 & -1.182 & 0.2480 \\
No. of branches & Quadratic & -0.8634 & \\
Growth rate & - & & & \\
\hline
\end{tabular}

Analyses were carried out separately for each environment, and each included linear and quadratic terms.

Standardized regression coefficients are reported.

Boldface indicates significant coefficients according to a sequential Bonferroni correction that accounts for multiple simultaneous tests across treatments.

(favouring decrease) as well as stabilizing selection on growth rate. We also found a significant stabilizing selection component on the plasticity of growth rate: in Fig. 1e most families with high fitness are clearly characterized by slow growth, although they are spread along a wide range of values and the family with the slowest growth rate has only intermediate fitness (Landsberg).

The within-treatment analyses present a much simpler picture (Table 3). Under low nutrients, there is a significant directional selection for increased height. Under high nutrients, the directional selection for increase in height is even stronger, and is accompanied by directional selection for reduced leaf number and by disruptive selection for flowering time.

The principal components analysis on genotypic means allows description of the multivariate plasticity of $A$. thaliana (Table 4). The first two eigenvectors cumulatively explained about 70 per cent of the total variance. The first principal component was defined primarily by the elevations of the reaction norms for all traits: average height and branching were positively correlated with each other and negatively correlated with leaf number, flowering time and growth rate. The second principal component was dominated by the plasticities of the traits, with growth rate plasticity negatively related to the plasticities of other traits. Regression analyses of the effect on fitness of the score of the families on each component (Table 4b) revealed highly significant directional selection for an increase in PC-1 (i.e. on the traits means).

\section{Discussion}

Variability in reaction norms to nutrients availability

There is a high degree of genetic variation, at both the population and the family levels, for parameters describing reaction norms to nutrient availability in $A$. thaliana. The analysis of variance showed that most variation was for the elevation of the reaction norm, with genetic variability in plasticity present for flowering time and for branching. The plots of reaction norms and the computation of interenviron- 
Table 4 (a) Principal components analysis for Arabidopsis thaliana based on family means, including the acrossenvironment mean and slope of the five characters assumed to influence fitness. (b) Multiple regressions relating genotypic scores on the first two principal components to across-environment fitness

\begin{tabular}{|c|c|c|c|c|}
\hline $\begin{array}{l}\text { (a) } \\
\text { Variable }\end{array}$ & Attribute & Explained variance: & $\begin{array}{r}\text { PC-1 } \\
39.5\end{array}$ & $\begin{array}{l}\text { PC-2 } \\
30.6\end{array}$ \\
\hline \multirow[t]{2}{*}{ No. of leaves } & Mean & & -0.29 & +0.15 \\
\hline & Plasticity & & -0.16 & +0.28 \\
\hline \multirow[t]{2}{*}{ Flowering time } & Mean & & -0.45 & +0.09 \\
\hline & Plasticity & & +0.01 & +0.48 \\
\hline \multirow[t]{2}{*}{ Height } & Mean & & +0.48 & +0.02 \\
\hline & Plasticity & & +0.14 & +0.52 \\
\hline \multirow[t]{2}{*}{ No. of branches } & Mean & & +0.43 & +0.21 \\
\hline & Plasticity & & +0.16 & +0.45 \\
\hline \multirow[t]{2}{*}{ Growth rate } & Mean & & -0.45 & +0.12 \\
\hline & Plasticity & & +0.15 & -0.36 \\
\hline (b) & Regression & Standard & & \\
\hline Trait & coefficient & error & $t$-ratio & $P$ value \\
\hline PC-1, linear & +0.1165 & 0.0122 & +9.55 & 0.0001 \\
\hline PC-1, quadratic & +0.0205 & 0.0094 & +2.18 & 0.0359 \\
\hline PC-2, linear & +0.0031 & 0.0289 & +0.11 & 0.9139 \\
\hline PC-2, quadratic & -0.0091 & 0.0089 & -1.02 & 0.3130 \\
\hline
\end{tabular}

(a) Weights of the original variables are reported for the first two eigenvectors.

Boldface indicates on which component each variable has the higher weight.

(b) Boldface indicates coefficients significant according to a sequential Bonferroni correction.

ment broad sense genetic correlations pointed to a marked difference between flowering time and growth rate on one hand, and number of leaves, number of branches and plant height on the other. The 37 reaction norms for the first two traits showed high interenvironmental genetic correlations. This is usually interpreted as a constraint on further evolution of the reaction norms (Via, 1993). Any selective pressure that would be directed toward modifying the phenotypic expression in one environment without altering it in the second one (or pushing it in the opposite direction), should encounter resistance because of reduced genetic variation. On the other hand, the scatter of reaction norms for height, branching and especially leaf number, was such that the corresponding genetic correlations were very low. Should selection favour increased or decreased plasticity for any of these traits, we would expect a quick response by a population constituted of the families examined here (de Jong, 1995; van Tienderen \& Koelewijn, 1994).

Most previous studies of $A$. thaliana have found plasticity and genetic variation for plasticity to nutrient availability in several characters. Pigliucci et al. (1995a) found genetic variation for plasticity to nutrients at the population level; the multivariate relationships among various traits, as assessed by path analysis, were markedly population- and treatment-specific. Zhang \& Lechowicz (1994) detected significant correlations among time of flowering, plasticity of flowering to nutrients and reproductive fitness in a study conducted on 13 populations. An appropriate response to nutrients in the soil might dramatically affect the population dynamics of $A$. thaliana, as suggested by Thompson (1994). A reasonable expectation is that plants growing under higher nutrients should become larger and increase both vegetative and reproductive outputs, which we found under our experimental conditions. However, under field conditions the density of conspecifics is also a determining factor (Myerscough \& Marshall, 1973; Thompson, 1994). The interactions between these two factors should therefore be investigated further under both field and controlled conditions.

It is worth noting that Landsberg, usually considered as 'wild-type' $A$. thaliana for experiments in molecular and developmental biology (Meyerowitz, 1989 ) is in fact atypical of the sample of families 
analysed here for number of leaves (very low), flowering time (very early) and growth rate (very slow). It is, on the other hand, characterized by median values of height and number of branches. It tends not to be plastic for any of the traits examined in the nutrient treatments; this is true for some but not all natural populations (Pigliucci \& Schlichting, 1995). Any future ecological/evolutionary interpretations of physiological-molecular analyses need to take Landsberg's derived phenotype and lack of plasticity into consideration.

\section{Relationships between reaction norms and fitness}

The problem of the determinants of fitness in heterogeneous environments has been at the centre of a number of theoretical as well as experimental papers (Levins, 1963; Weis \& Gorman, 1990; Bell, 1992; Karron \& Marshall, 1993). The very concept of fitness is a rather controversial one (review in Ollason, 1991), and Alexander (1991) and Lauder et al. (1993) have pointed out the complexity of the relationship between measurable performances or adaptation in the sense of the product of historical processes, and fitness. Here we do not make any attempt to relate directly fitness in these families as observed under controlled conditions to past selective pressures on the reaction norms. To do that meaningfully would require substantial knowledge of the habitat distribution and of the fitness functions in the field, knowledge that is currently lacking (but see Weis \& Gorman, 1990). On the other hand, we maintain that an understanding of the effects of several characters on reproductive output when only one or few environmental variables are altered at a time, must be part of the ultimate solution to the general problem of understanding the relationships between phenotype and fitness in changing conditions.

We found evidence that under the specific conditions of this experiment the parameters describing the reaction norms (i.e. elevation and slope) of several characters do affect reproductive fitness as measured by fruit output in this species. The most prevalent mode of selection was directional on trait means, but instances of stabilizing and disruptive selection, as well as selection on plasticity, were also found. When we analysed selection pressures within treatments, a much simpler scenario emerged, mostly emphasizing directional selection. This simplification is no doubt related to the fact that these regressions neglect to account for any interaction between populations (or families) and environment. Even though results obtained from within-environment analyses can be translated into their across-environment equivalents (de Jong, 1995) this process is not mathematically straightforward. Therefore, we suggest adopting both approaches for a better understanding of selection regimes. Clearly, estimates of the relative frequencies of different natural environments are vital if we wish to translate estimates of selection under controlled conditions to the field. This type of information is persistently lacking in most ecological genetics studies.

When we considered the multivariate phenotype of $A$. thaliana, we were able to identify a major axis of variation characterized by the reaction norms' elevations and a second axis defined by the plasticities. The sign of the weights on PC-1 (reaction norms' elevations) point to the existence of two sets of covarying characters. Because the PCA was performed on the family means, this suggests a genetic constraint among the 37 families analysed: taller plants with increased branching (both traits favoured by selection) would also be characterized by lower leaf number, earlier flowering and a slower growth rate (all of which would also be favoured under the conditions tested in our experiment). In fact, the linear regression coefficient on the first principal component was highly significant. However, this scenario allows us to predict response to selection only in the short term (Schlichting \& Pigliucci, 1995): eventually, biochemical or physical constraints must limit the lowest possible leaf number or the fastest phenology that can be achieved, especially while simultaneously trying to increase branching and plant size. The structure of the second eigenvector is equally interesting, relating the plasticities of all five traits. However, we did not detect significant selection on this component. This might have two causes. (i) Insufficient power of the multiple regression to detect significance on a variable summarizing 30 per cent of the total variance (and calculated on the family means). (ii) Only the plasticities of two traits (branching and growth rate) were under selection at the univariate level and these are negatively related on PC-2: this inverse relationship might effectively cancel any selective force acting on the two independently, and represents a prime example of a potential constraint on the evolution of plasticity.

\section{Acknowledgements}

We thank the following persons for reading previous versions of this manuscript and/or for comments on the topics here covered: Guido Barbujani, Yves Carrière, Kittie Donohue, Susan Dudley, Monica 
Geber, Kent Holsinger, Cynthia Jones, Phil dilorio, Annie Schmitt and John Silander. Thanks to Lavanya Gullapalli for assistance with the measurements. This work was supported by NSF dissertation improvement grant DEB-9122762 and by a Sigma-Xi Grant-in-Aid to MP, and by NSF grant DEB-9220593 to CDS.

\section{References}

ABBotT, R. J. AND GOMEs, M. F. 1989. Population genetic structure and outcrossing rate of Arabidopsis thaliana (L.) Heynh. Heredity, 62, 411-418.

ALEXANDER, R. McN. 1991. Apparent adaptation and actual performance. Evol. Biol., 25, 357-373.

BELL, G. 1992. The ecology and genetics of fitness in Clamydomonas. V. The relationship between genetic correlation and environmental variance. Evolution, 46, $561-565$.

BRADSHAW, A. D. 1965. Evolutionary significance of phenotypic plasticity in plants. Adv. Genet., 13, 115-155.

DE JONG G. 1994. The fitness of fitness concepts and the description of natural selection. Q. Rev. Biol., 69, 3-29.

DE JONG, G. 1995. Phenotypic plasticity as a product of selection in a variable environment. Am. Nat., 145, 493-512.

GEBER, M. A. 1990. The cost of meristem limitation in Polygonum arenastrum: negative genetic correlations between fecundity and growth. Evolution, 44, 799-819.

JONES, M. E. 1971. The population genetics of Arabidopsis thaliana. II. Population structure. Heredity, 27, 51-58.

KARRON, J. D. AND MARSHALL, D. L. 1993. Effects of environmental variation on fitness of singly and multiply sired progenies of Rhaphanus sativus (Brassicaceae). Am. J. Bot., 80, 1407-1412.

LACEY, E. P., REAL, L. ANTONOVICS, J. AND HAECKEL, D. G. 1983. Variance models in the study of life histories. $A m$. Nat., 122, 114-131.

LAUDER, G. V., LEROI, A. M. AND ROSE, M. R. 1993. Adaptations and history. Trends Ecol. Evol., 8, 294-297.

LEVINS, R. 1963. Theory of fitness in a heterogeneous environment. II. Developmental flexibility and niche selection. Am. Nat., 47, 75-90.

MEyerowitz, E. M. 1989. Arabidopsis, a useful weed. Cell, 56, 263-269.

MYERSCOUGH, P. J. AND MARShall, J. K. 1973. Population dynamics of Arabidopsis thaliana (L.) Heynh. strain 'Estland' at different densities and nutrient levels. New Phytol., 75, 595-617.

OLlason, J. G. 1991. What is this stuff called fitness? Biol. Phil., 6, 81-92.

Pigliucci, M. AND SChlichting, C. D. 1995. Reaction norms of Arabidopsis (Brassicaceae). III. Response to nutrients in 26 populations from a worldwide collection. Am. J. Bot., 82, 1117-1125.
PIGLIUCCI, M., WHITTON, J. AND SCHLICHTING, C, D. 1995a Reaction norms of Arabidopsis. I. Plasticity of characters and correlations across water, nutrient and light gradients. J. Evol. Biol., 8, 421-438.

PIGliUCCI, M., SCHLiChTiNG, C. D. AND WhitTon, J. 1995b. Reaction norms of Arabidopsis. II. Response to stress and unordered environmental variation. Funct. Ecol., 9, 537-547.

RAUSHER, M. D. 1992. The measurement of selection on quantitative traits: biases due to environmental covariances between traits and fitness. Evolution, 46, 616-626.

ROFF, D. A. AND PREZIOSI, R. 1994. The estimation of the genetic correlation: the use of the jackknife. Heredity, 73, 544-548.

SAS, 1990. SAS/STAT User's guide. SAS Institute, Cary, NC.

SCHEINER, s. M. 1993. Genetics and evolution of phenotypic plasticity. Ann. Rev. Ecol. Syst., 24, 35-68.

schlichting, C. D. 1986. The evolution of phenotypic plasticity in plants. Ann. Rev. Ecol. Syst., 17, 667-693.

SChlichting, C. D. AND PIGliuCCI, M. 1993. Control of phenotypic plasticity via regulatory genes. Am. Nat., $142,366-370$.

SCHLICHTING, C. D. AND PIGLIUCCI, M. 1995. Gene regulation, quantitative genetics and the evolution of reaction norms. Evol. Ecol., 9, 154-168.

schmalhausen, I. I. 1949. Factors of Evolution. Chicago University Press, Chicago.

SERVICE, P. M. AND ROSE, M. R. 1985. Genetic covariation among life-history components: the effect of novel environments. Evolution, 39, 943-945.

SHAW, R. G. 1991. The comparison of quantitative genetic parameters between populations. Evolution, 45 143-151.

Sultan, s. E. 1987. Evolutionary implications of phenotypic plasticity in plants. Evol. Biol., 21, 127-178.

THOMPSON, L. 1994. The spatiotemporal effects of nitrogen and litter on the population dynamics of Arabidopsis thaliana. J. Ecol., 82, 63-68.

VAN TIENDEREN, P. H. AND KOELEWIJN, H. P. 1994. Selection on reaction norms, genetic correlations and constraints. Genet. Res., 64, 115-125.

VIA, s, 1993. Adaptive phenotypic plasticity: target or by-product of selection in a variable environment? $\mathrm{Am}$. Nat., 142, 352-365.

WE1S, A. E. AND GORMAN, w. L. 1990. Measuring selection on reaction norms: an exploration of the Eurosta-Solidago system. Evolution, 44, 820-831.

WESTERMAN, J. M. AND LAWRENCE, M. J. 1971. Genotypeenvironment interaction and developmental regulation in Arabidopsis thaliana. I. Inbred lines; description. Heredity, 25, 609-627.

ZHANG, J. AND LECHOWICZ, M. J. 1994. Correlation between time of flowering and phenotypic plasticity in Arabidopsis thaliana. Am. J. Bot., 81, 1336-1342. 